INPLASY

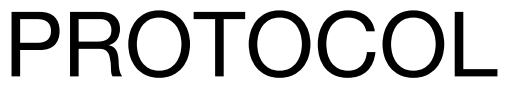

To cite: Wu et al. The effect of cryotherapy for patients with chemotherapy-induced peripheral neuropathy: A systematic review and metaanalysis. Inplasy protocol 202150042. doi: 10.37766/inplasy2021.5.0042

Received: 10 May 2021

Published: 11 May 2021

Corresponding author: Binxi Wu

490303239@qq.com

Author Affiliation:

Guangdong Pharmaceutical University

Support: None.

Review Stage at time of this submission: Preliminary searches.

Conflicts of interest: None declared.

\section{The effect of cryotherapy for patients with chemotherapy-induced peripheral neuropathy: A systematic review and meta-analysis}

\author{
Wu, B1; Chen, G²; He, Z3; Wang, Q4 .
}

Review question / Objective: To demonstrate the effectiveness of cryotherapy for chemothrapy-induced peripheral neuropathy through published clinical trials.

Condition being studied: Accompanying acute pain in peripheral nerves, chemotherapy-induced peripheral neuropathy (CIPN), which is defined as a adverse reaction caused by drugs for anti-cancer like oxaliplatin or paclitaxel, forces patients to quit chemotherapy. Amid a few therapies for CIPN (drug intervene or non-drug intervene), cryotherapy shows its effectiveness in some published RCTs. In this mataanalysis, we are to demonstrate effectiveness of cryotherapy for CIPN by existed clinical trials.

INPLASY registration number: This protocol was registered with the International Platform of Registered Systematic Review and Meta-Analysis Protocols (INPLASY) on 11 May 2021 and was last updated on 11 May 2021 (registration number INPLASY202150042).

\section{INTRODUCTION}

Review question / Objective: To demonstrate the effectiveness of cryotherapy for chemothrapy-induced peripheral neuropathy through published clinical trials.
Condition being studied: Accompanying acute pain in peripheral nerves, chemotherapy-induced peripheral neuropathy (CIPN), which is defined as a adverse reaction caused by drugs for anticancer like oxaliplatin or paclitaxel, forces patients to quit chemotherapy. Amid a few 
therapies for CIPN (drug intervene or nondrug intervene), cryotherapy shows its effectiveness in some published RCTs. In this mata-analysis, we are to demonstrate effectiveness of cryotherapy for CIPN by existed clinical trials.

\section{METHODS}

Participant or population: Patients with chemotherapy-induced peripheral neuropathy.

Intervention: Cryotherapy.

Comparator: Compression therapy; no intervenes as control group.

Study designs to be included: Clinical trials.

Eligibility criteria: The authors did not describe eligibility criteria.

Information sources: PubMed, Cochrane Library, EMBASE, Chinese Biological Medicine Database, China National Knowledge Infrastructure, Wanfang Database, and the Chinese Scientific Journal Database.

Main outcome(s): Cryotherapy reduces neuropahty symptoms.

Quality assessment / Risk of bias analysis: The Cochrane Collaboration's risk of bias tool.

Strategy of data synthesis: Synthetic data comes from Reciew Manager.

Subgroup analysis: Participants in compression therapy; participants in cryotherapy.

Sensitivity analysis: To conduct sensitivity analysis by using Review Manager.

Country(ies) involved: China.

Keywords: cryotherapy; cold therapy; frozen gloves; chemotherapy-induced peripheral neuropathy.
Contributions of each author:

Author 1 - Binxi Wu.

Author 2 - Guien Chen.

Author 3 - Ziji He.

Author 4 - Qiuhong Wang. 